\title{
A MEMS-BASED TUNABLE RF CHANNEL-SELECTING SUPER-REGENERATIVE TRANSCEIVER FOR WIRELESS SENSOR NODES
}

\author{
Tristan O. Rocheleau, Thura Lin Naing, Jalal Naghsh Nilchi, and Clark T.-C. Nguyen \\ University of California, Berkeley, USA
}

\begin{abstract}
An electromechanical circuit comprising a capacitive-gap RF MEMS resonator embedded in a positive feedback loop with an ASIC transimpedance amplifier has demonstrated a first fullyfunctional MEMS-based tunable RF channel-selecting radio transceiver employing a super-regenerative reception scheme. Unlike previous super-regenerative receivers, this rendition harnesses the exceptionally high $Q$ around 100,000 and voltage-controlled frequency tuning of its capacitive-gap transduced disk resonator to enable selection of any one of among twenty $1-\mathrm{kHz}$-wide RF channels over an $80-\mathrm{kHz}$ range, while rejecting adjacent channels and consuming under $490 \mu \mathrm{W}$. These are well suited to the target wireless sensor node application, for which low-power and simplicity trump transmission rate. Electrical stiffness-based frequency tuning also allows this same device to operate as an FSK transmitter, making it a complete transceiver in one simple device. Finally, the geometric flexibility of capacitive-gap transduced resonator design should permit a large range of usable RF frequencies, from the presently demonstrated $60.6-\mathrm{MHz}$ VHF, all the way up to UHF.
\end{abstract}

\section{INTRODUCTON}

The field of RF-MEMS has thus far improved many aspects of wireless communication, with commercially available products ranging from on-chip MEMS devices providing compact and low phase-noise reference oscillators $[1,2]$, to band-selecting RF front-end duplexers [3]. Greater potential remains, however, if the high quality factor and CAD-definable frequency possible in capacitive-gap MEMS can be harnessed to achieve a true channel-selection scheme.

In particular, MEMS-based communication offers a viable path towards meeting the ultra-low-power requirements demanded by wireless sensor networks. Tiny, low-cost wireless motes, where onboard sensors collect and transmit data through a mesh network while operating only on scavenged or battery power, stand to revolutionize data collection in applications ranging from industrial or environmental monitoring to biomedical imaging. While significant advances have been made towards such goals [4], the power consumption of current conventional-technology efforts are still in the milliwatt range, in excess of that needed for true set-and-forget sensor nodes.

Capacitive-gap MEMS devices have emerged as one option that provides the high quality factors $(Q)$ that make possible the narrow bands required to greatly lower power. With $Q>40,000$ even at $\mathrm{GHz}$ frequencies [5], such resonators easily allow for the tightly-spaced $\mathrm{kHz}$-frequency bands ideal for sensor nodes, without requiring the processing and power-hungry spread-spectrum approaches currently used. Indeed, power consumption of oscillators constructed from such resonators has been demonstrated below $100 \mu \mathrm{W}$ [1], even in the face of modest resonator impedance in the $\mathrm{k} \Omega$-range. These are ideally suited to the target wireless sensor node application, for which low-power and simplicity trump transmission rate.

In this paper, one such electromechanical circuit comprising a capacitive-gap RF MEMS resonator ( $c f$. Fig.1) embedded in a positive feedback loop with an Application-Specific Integrated Circuit (ASIC) Trans-Impedance Amplifier (TIA) has demonstrated a fully-functional MEMS-based tunable RF channel-selecting radio transceiver employing a super-regenerative reception scheme.

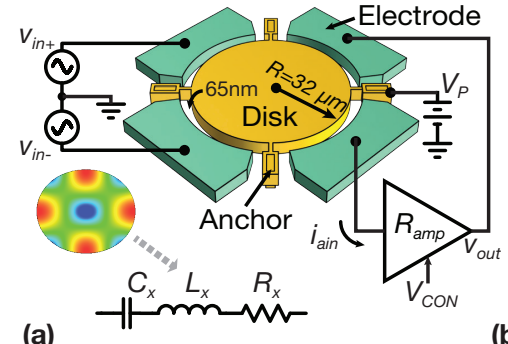

(a)
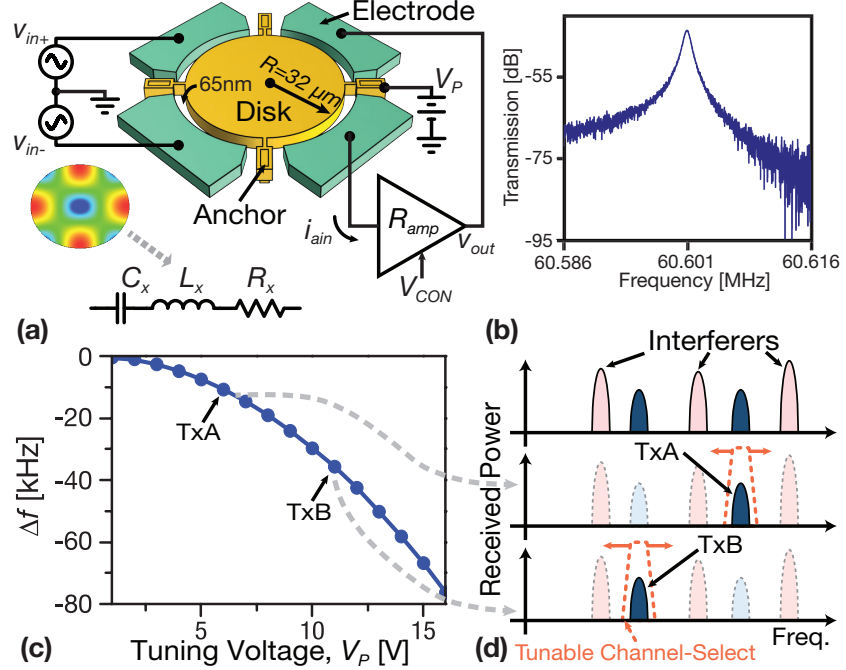

Figure 1. Perspective-view schematic (a) of the micromechanical disk resonator circuit used in this work. (b) presents the high $Q \sim 100,000$ resonator frequency response, which may be tuned as in (c) via negative electrical stiffness with applied voltage to select one of several communication channels as illustrated in (d).

Unlike previous super-regenerative receivers $[6,7]$, this rendition harnesses the high $Q$ filtering and voltage-controlled frequency tuning [8] of its capacitive-gap transduced disk resonator to enable selection of $1-\mathrm{kHz}$-wide RF channels across a $80-\mathrm{kHz}$ range, as in Fig. 1(c,d). Furthermore, the split electrode resonator design made possible here isolates the loop amplifier from the antenna, greatly relaxing circuit linearity requirements and eliminating the need for an isolation amplifier, thus saving considerable power.

This electrical stiffness-based tuning additionally allows the same MEMS-ASIC system to operate as an FSK transmitter, enabling a complete transceiver in one simple device. Operated as a closedloop oscillator with FSK modulation enabled via an applied voltage on the MEMS input electrodes, this transmitter offers direct carrier generation at the RF frequency of interest without the power-hungry complexity of previous PLL-based MEMS transmitters [9].

\section{THE REGENERATIVE TRANSCEIVER}

Fig. 2 presents the system-level design used for the superregenerative receiver in this work, while Fig. 3 illustrates typical operation. This super-regenerative system identifies an incoming ' 1 ' or ' 0 ' by measuring the rate at which oscillation grows in a positive feedback circuit. In this rendition, the absence of received antenna signal power in the resonance passband results in a slow rise in oscillation amplitude, which indicates a ' 0 '. On the other hand, with received power on resonance, signal couples into the positive feedback loop, speeding up rise time to indicate a ' 1 '. Operated in such a fashion, this regenerative receiver forms a Binary FrequencyShift Keying (BFSK) demodulator, where the FSK 'mark' and 'space' correspond to on and off-resonance signals, respectively.

To generate a stream of received bits, a periodic quench signal $V_{C O N}$ restarts oscillation, allowing each bit to be decoded in a separate, short oscillation growth as seen in Fig. 3(b). Fig. 3(c) 


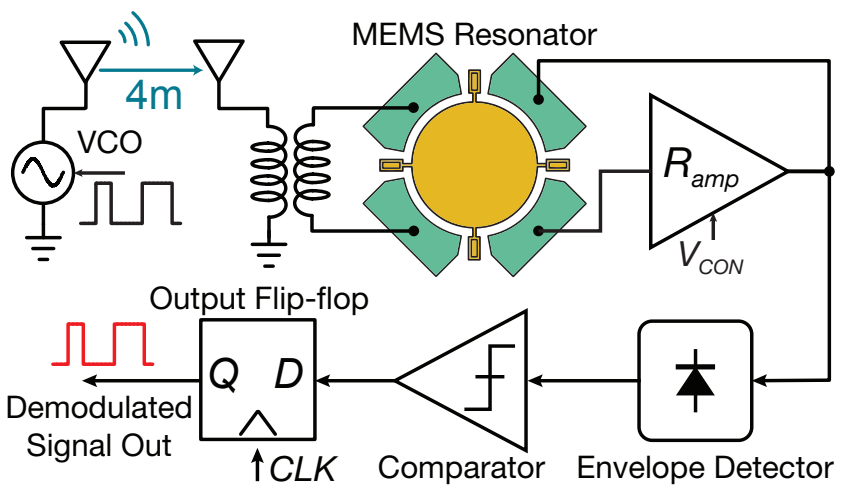

Figure 2. Schematic of the regenerative MEMS radio transceiver configured in receive-mode. Here, the MEMS resonator applies the needed channel-selecting filter to RF signals picked up by the antenna. The ASIC amplifier regeneratively amplifies this weak received in-band signal to produce a growing oscillation output, periodically quenched via $V_{C O N}$. An envelope detector, comparator and flop-flop latch recovers the original transmitted digital data.
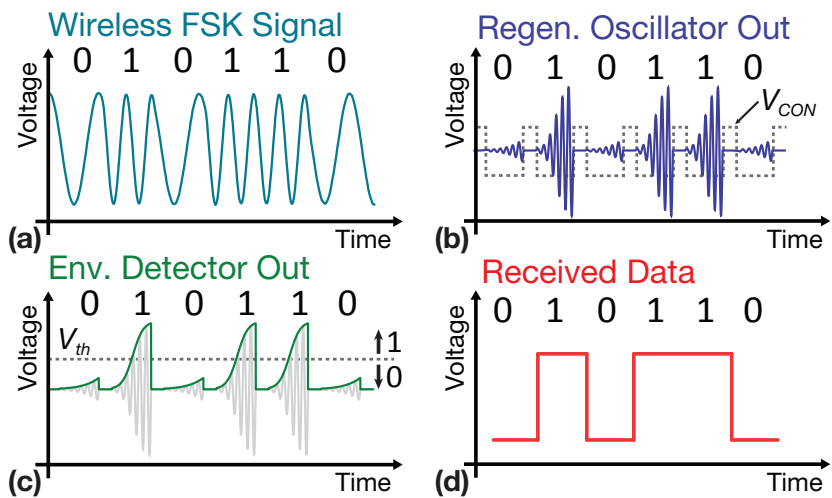

Figure 3. Conceptual operation of the regenerative receiver. The BFSK modulated and transmitted waveform of (a) gives rise to the periodically restarted oscillation of (b), where the on-resonance signal generates faster oscillation growth. Here, amplifier output drops to zero when $V_{\text {con }}$ is raised, though resonator motion decays with a time constant based on resonator $Q$. Finally, envelope amplitudes which exceed $V_{\text {th }}$ in (c) allow discrimination of ' 0 's and ' 1 's, recovering the original data in (d).

presents the envelope of the periodic oscillation growth produced by the envelope detector of Fig. 2. The output of this envelope detector feeds a comparator, the output of which in turn is latched into a flipflop to recover the original transmitted data (Fig. 3(d)).

With the high $Q$ of the MEMS resonators, this receiver isolates a single narrow channel while rejecting signals in nearby channels, spaced here at $4 \mathrm{kHz}$ apart. Because the amplifier in such a design is isolated from the input antenna with only on-resonance signals passed through the MEMS resonator, out-of-channel interferers are blocked. This marks a significant improvement over previous regenerative receiver architectures [6], where loop amplifiers must handle any spurious signal received by the antenna without causing excess intermodulation. Here, the filtering of any such interferers greatly relaxes the linearity, and hence power consumption, of the amplifier.

Meanwhile, with voltage tunable via electrical-stiffness, such MEMS oscillators may also be configured as frequency-modulated elements, offering a uniquely simple, continuous-phase FrequencyShift Keying transmitter. Such operation can be enabled using the same circuit as used for receive-mode, but with amplifier gain held

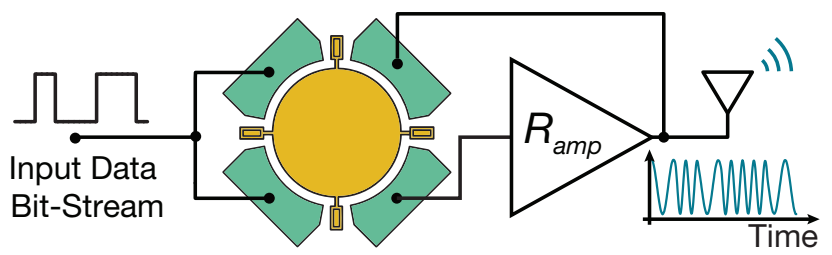

Figure 4. Schematic of the MEMS radio transceiver configured in transmit-mode where demodulating circuitry is switched out for an FSK modulating input to the capacitive-gap disk. With TIA gain no longer quenched, a continuous RF carrier is generated.

constant to generate a continuous RF carrier as shown in Fig. 4. Data to be transmitted may be applied to the input MEMS electrodes to generate FSK modulation, while the output of the amplifier is wired to the antenna.

\section{Sustaining Amplifier Design}

Fig. 5 presents the transimpedance amplifier ASIC used to achieve regenerative amplification (in receive mode, $c f$. Fig. 2) and the FSK modulated carrier (in transmit mode, $c f$. Fig 4). This circuit, fabricated in a $0.35 \mu \mathrm{m}$ CMOS process, consists of a differential CMOS amplifier with output taken from one side, with the other side connected in shunt-shunt feedback to achieve a $0^{\circ}$ phase shift from input to output while realizing controllable gain via a feedback resistance. Transistors $M_{1}-M_{4}$ comprise the basic differential pair biased by a Common-Mode FeedBack (CMFB) circuit; $M_{R F}$ serves as the voltage controllable feedback resistor to allow control of the total transimpedance gain via input bias voltage $V_{C O N}$.

With transresistance gain $R_{\text {amp }}$ sufficient to overcome resonator losses, the oscillator loop amplitude rises exponentially with time constant given by

$$
\tau=\frac{2 L_{x}}{R_{a m p}+R_{x}}
$$

where $R_{x}$ and $L_{x}$ are the equivalent circuit model elements for the MEMS resonator tank shown in Fig. 1(a). An on-resonance signal reduces the time required to rise to the ' 1 '-bit threshold amplitude in two ways: first, the drive signal is resonantly enhanced to produce a resonator starting amplitude at the beginning of each oscillation cycle much larger than the background thermal noise, and second, this input signal continues to drive the resonator to increased amplitude during oscillation startup.

As with any filter, the bandwidth of the filter limits the possible data transmission rate. Here, such a limit is enacted by the decay time required for the resonator to reach a low amplitude of motion following a ' 1 '. If the amplifier quench time falls short of the decay time of the resonator (here a long $0.5 \mathrm{~ms}$ due the high resonator $Q$ ), oscillation will restart quickly even in the absence of an input signal, preventing detection of subsequent ' 0 's.

\section{MEMS Resonator}

To meet the filtering requirements of channel-select radio applications, the MEMS resonators used must possess both a useful RF operating frequency and sufficient $Q$ to achieve the narrow $\sim 1$ $\mathrm{kHz}$ bandwidths desired for the sensor node application. To this end, the disk resonators depicted in Fig. 1(a) and Fig. 2 are quite suitable, with the added capability to accurately define multiple unique frequencies on the same die via only CAD layout, thus expanding possible operating frequency beyond that achievable from electrical stiffness tuning alone. The base device comprises a 2 $\mu \mathrm{m}$-thick polysilicon disk supported by beams at quasi-nodal points and coupled along its sidewalls to input-output electrodes by tiny 

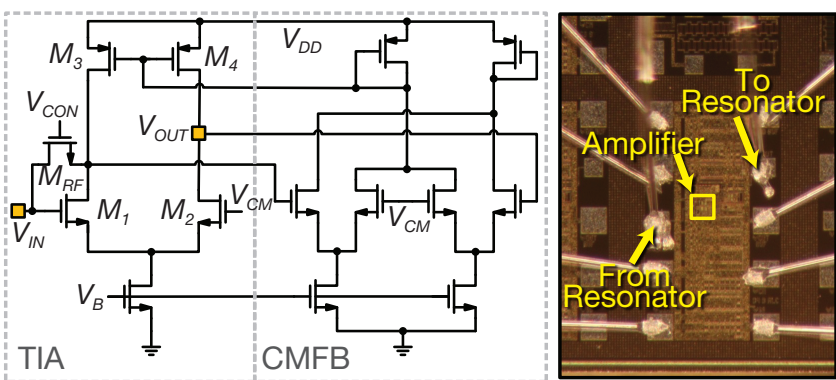

Figure 5. Schematic of the transimpedance amplifier circuit (left) and die photo (right). Bondwires connect the MEMS resonator between nodes $V_{\text {in }}$ and $V_{\text {out }}$ as in Fig. 2 to generate a closed oscillator loop with loop gain controlled by voltage applied to $V_{C O N}$.

$65 \mathrm{~nm}$ capacitive gaps. To drive the resonator into motion, a bias voltage $V_{P}$ on the disk structure combines with a differential ac drive voltage applied to input electrodes to produce forces across the input electrode-to-resonator gaps that, at resonance, excite the compound $(2,1)$ mode shape, shown in the inset of Fig. 1(a). The frequency of resonance is given by [10]:

$$
f_{\text {nom }}=\frac{K}{R} \sqrt{\frac{E}{\rho(2+2 \sigma)}}
$$

where $R$ is the disk radius, $K$ a material-dependent constant equal to 0.373 for polysilicon, and $E, \sigma$, and $\rho$ are the Young's modulus, Poisson ratio, and density of the polysilicon material, respectively.

In such resonators, a position-dependent electrical force across the capacitive gap additionally gives rise to the required electrical stiffness effect and resultant resonance frequency shift [10], given by:

$$
k_{e}=-\alpha \frac{\varepsilon_{0} A V_{P}^{2}}{d^{3}} ; \quad f_{0}=f_{n o m}\left[1+\frac{k_{e}}{k_{m}}\right]^{\frac{1}{2}}
$$

where $\varepsilon_{o}$ is the vacuum permittivity, $A$ is the resonator-electrode overlap area, $V_{P}$ is the voltage placed across the gap, $d$ is gap width, and $\alpha$ is a dimensionless constant based on mode and electrode shape, equal to 0.637 for the design used here.

\section{EXPERIMENTAL REALIZATION}

Using Eqn. (3), MEMS resonators were designed for operation at $60.6 \mathrm{MHz}$ with a disk radius of $32 \mu \mathrm{m}$. Fig. 6 summarizes the surface micromachining process used for fabrication, based on the same process as [11]. Here, phosphorus-doped polysilicon deposited via low-pressure chemical-vapor deposition (LPCVD) at $615{ }^{\circ} \mathrm{C}$ provided all resonator structure, electrode and electrical interconnect material. A high-temperature oxide (HTO) sidewall sacrificial deposition defined the $65 \mathrm{~nm}$ resonator-to-electrode gaps. Two chemical-mechanical polishing (CMP) steps, one before structural polysilicon deposition and patterning and the other after the electrode polysilicon deposition, provided planar surfaces desired for precise lithography, as well as removed electrode-disk overhangs that can otherwise cause pull-in and device failure at low bias voltages. Following fabrication, structures were released in $49 \% \mathrm{HF}$ to yield the final device imaged in the SEM of Fig. 6.

To construct a complete radio transceiver, released MEMS resonators are bondwired together with the CMOS ASIC described above and affixed to $\mathrm{PCB}$ circuits that provided all needed bias voltages as well as the simple off-chip RF diode detector and flipflop circuit used for data recovery. Fig. 7 presents this assembled MEMS transceiver mounted in a custom bell-jar setup to allow measurement in a $\mu$ torr vacuum. The hermetic feedthroughs seen on the right connect the radio to a simple antenna formed frome

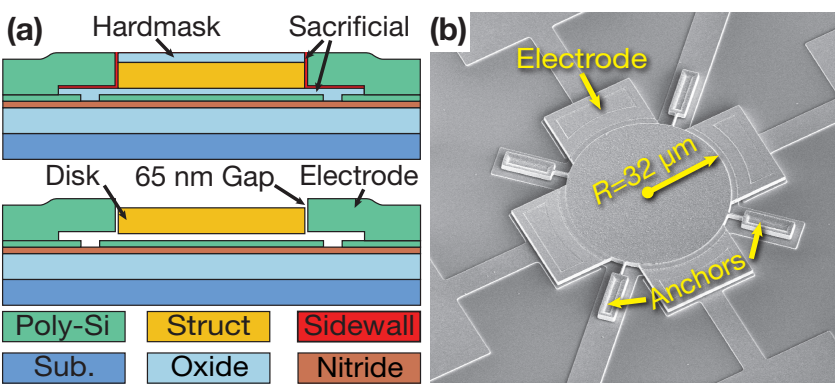

Figure 6. Cross sections (a) illustrating the MEMS resonators before (top) and after release in 49\% HF (bottom). (b) presents an SEM of one completed device used in this work.

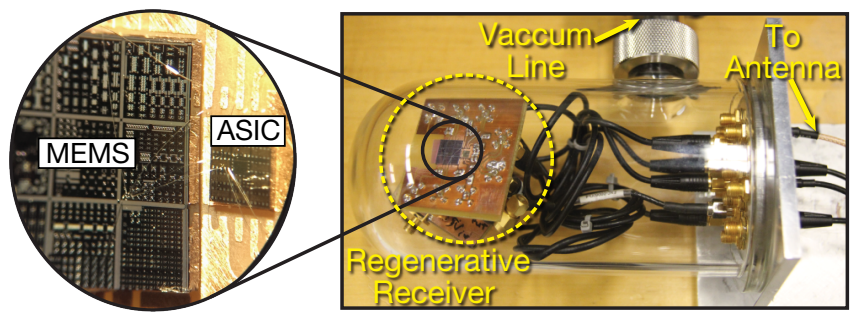

Figure 7. Measurement setup consisting of the regenerative transceiver dies mounted on a Printed Circuit Board (PCB) and measured in a bell-jar vacuum environment. The inset displays the MEMS and ASIC dies, both fabricated with many devices on a single die, one each of which is bondwired to the PCB.

$\sim 20 \mathrm{~cm}$ of wire. A bench-top frequency-synthesizer connected to a $2^{\text {nd }}$ antenna positioned $4 \mathrm{~m}$ from the receive antenna provided wirelessly transmitted test signals.

\section{Measurements}

Fig. 8 presents measured time-traces illustrating operation of the receiver circuit of Fig. 2. Here, a frequency synthesizer transmits the $-17 \mathrm{dBm}(20 \mu \mathrm{W})$ test signal modulated with the bit-stream of Fig. 8(a) using 1-kHz mark-space separation FSK at a data rate of $1 \mathrm{kbps}$. Gating the regenerative amplifier gain control $V_{C O N}$ at the receive data rate to periodically grow and quench oscillation produces the oscillation envelope of Fig. 8(c), generated from a receive antenna located at a distance of $4 \mathrm{~m}$ from the transmitter. Here, small in-band signals are regeneratively amplified to create the observed speed-up in oscillation amplitude growth, clearly distinguishing mark vs. space frequency of the transmitted signal. Fig. 8(d) presents the output of the comparator following the envelope detector, while Fig. 8(e) shows the final data latched into the output flip-flop, clearly recovering the original transmitted data from Fig. 8(a).

Despite the use of make-shift antennas, the performance is remarkable and demonstrates the sensitivity and selectivity of this capacitive-gap MEMS-based receiver, even in the face of typical interferers in an unshielded environment. To further gauge this ability to reject interference, Fig. 9 presents the measured receiver output with the transmitter detuned by $4 \mathrm{kHz}$ to a nearby channel and increased by $30 \mathrm{~dB}$, showing no detected signal, as it should.

Meanwhile, Fig. 10 demonstrates the BFSK-modulated output of the transmit-mode configuration in Fig. 4, where the TIA gain is no longer gated and the transmit data-stream is applied to the input electrodes. With the input data encoded in the $200 \mathrm{mV}$ swing seen in Fig. 10(a), the oscillation output (Fig. 10(b)) is seen to undergo a fast-response, continuous-phase FSK modulation, well-suited to the desired transmit function. 
(a) $\begin{array}{lllllllllllllllllll}0 & 1 & 0 & 1 & 1 & 0 & 1 & 1 & 0 & 1 & 1 & 0 & 1 & 0 & 0 & 1 & 1 & 1\end{array}$

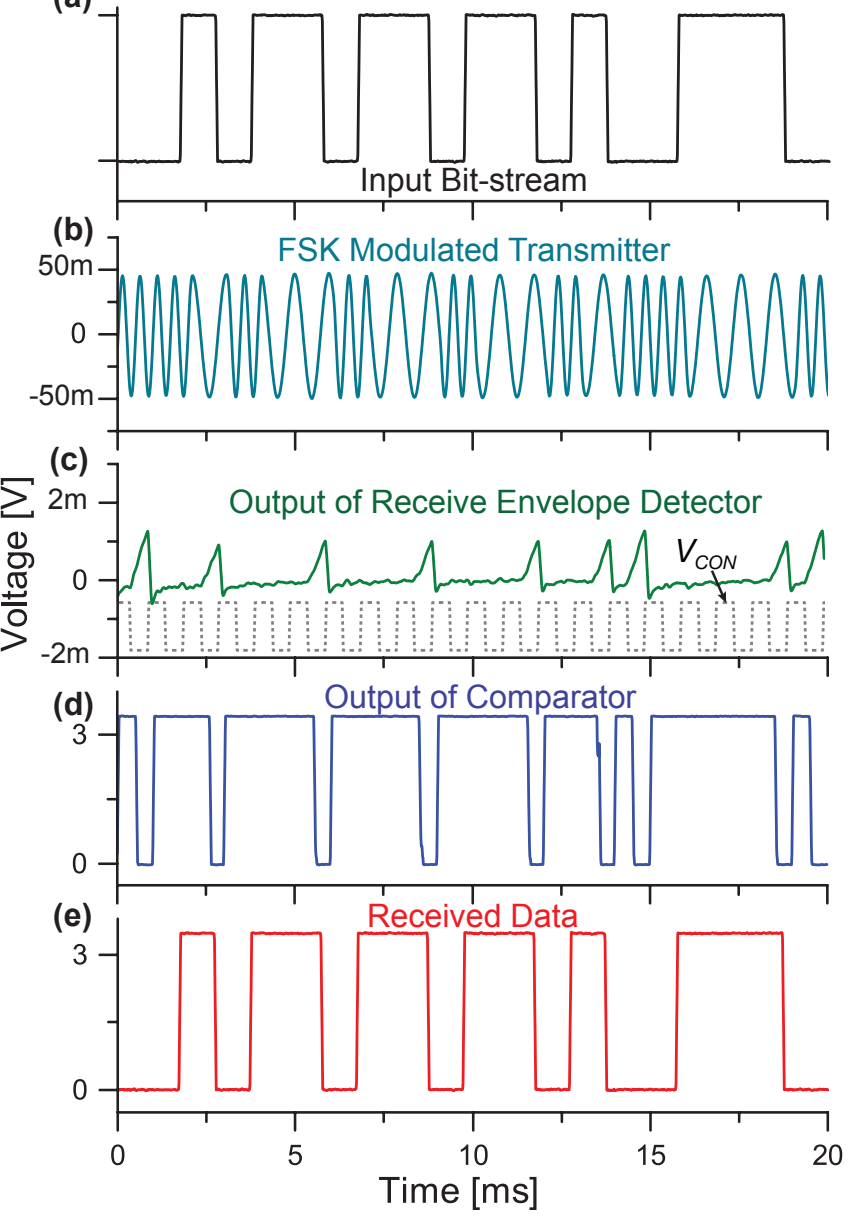

Figure 8. Measured receiver operation for a $1 \mathrm{kbps}$ bit-stream transmitted across a distance of $4 \mathrm{~m}$ as in Fig. 2 using a BinaryFSK modulated transmitter with $1 \mathrm{kHz}$ shift and output power of $-17 \mathrm{dBm}(20 \mu \mathrm{W})$. The transmitted data-stream (a) is BFSK encoded and transmitted on a $60.6 \mathrm{MHz} R F$ carrier (b) using a bench-top signal generator (shown mixed down to $2 \mathrm{kHz}$ ). Regenerative detection yields (c) amplitude following envelope detection; (d) shows resultant signal following comparator; and (e) presents the final recovered data-stream clocked by the output flip-flop. Here, the MEMS resonator is biased with $10.3 \mathrm{~V}$, and the ASIC is operated on a 2.1 V supply drawing $\sim 233 \mu \mathrm{A}$.

\section{CONCLUSIONS}

Using a periodically quenched transimpedance amplifier ASIC, the demonstrated MEMS-based circuit provides not only reliable selection and detection of individual narrow-band channels, but also FSK generation for transmission, and marks a first demonstration of an RF channel-select-capable MEMS radio transceiver. Additionally, a separate set of MEMS resonator electrodes for the antenna input and the amplifier loop shield the amplifier from out of band interferers. This greatly relaxes the amplifier linearity spec, which lowers its power consumption to enable a significant improvement over previous regenerative MEMS receiver topologies.

The demonstrated transceiver now offers a compelling new option for wireless sensor node devices. The power consumption here already offers significant improvement over previous sensor node systems [4], while the use of capacitive-gap MEMS devices provide extremely small size. Of course, transmit power above a few $\mu \mathrm{W}$ will require an additional power amplifier. For this, the narrow

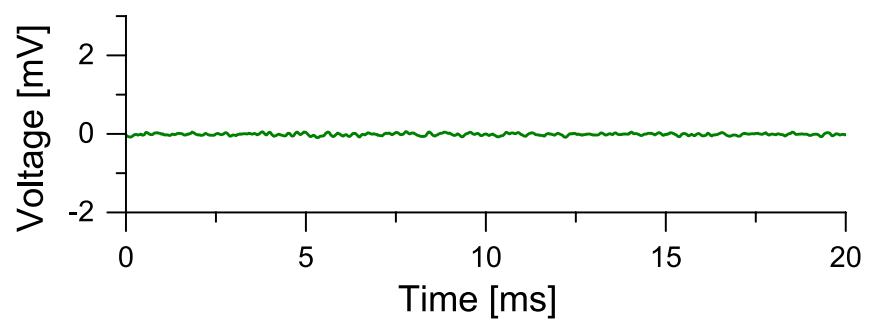

Figure 9. Measured regenerative receiver signal when transmitter is shifted by $4 \mathrm{kHz}$ to a near-by channel and increased $30 \mathrm{~dB}$ in power.

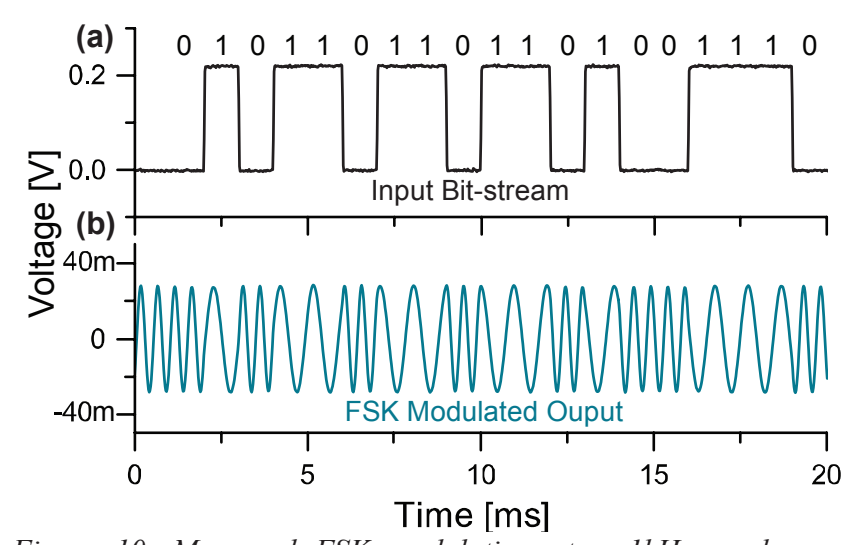

Figure 10. Measured FSK modulation at a $1 \mathrm{kHz}$ mark-space frequency separation generated using the transmit-mode configuration of Fig. 3. With the applied modulation signal (a), a continuous-phase output modulation is produced (b), shown here mixed down to $\sim 2 \mathrm{kHz}$ for presentation.

band and constant envelope of the FSK signal encourage the use of efficient RF amplifier topologies, and even opens the possibility of using the MEMS device itself as an efficient Class E amplifier [12]. If expectations for lowering power consumption to less than $100 \mu \mathrm{W}$ (such as has already been demonstrated in a similar MEMS oscillator design [1]) are on target, then true set-and-forget nodes may soon become possible, capable of operating for long periods on tiny on-board batteries or even just scavenged power.

Acknowledgment: This work was supported by DARPA.

\section{REFERENCES}

[1] T. L. Naing, et al., Proceedings, IFCS, 2013, pp. 562-565.

[2] H. Lee, et al., "Low Jitter and Temperature Stable MEMS Oscillators," Proceedings, IFCS, 2012, pp. 271-275.

[3] Avago, LTE Band 7 Duplexer, Part No. ACMD-6007

[4] J. M. Rabaey, et al., Computer, vol. 33, pp. 42-48, 2000.

[5] T. L. Naing, et al., Proceedings, IFCS, 2012, pp. 276-281.

[6] B. Otis, et al., Proceedings, ISSCC, 2005, pp. 396-606 Vol. 1.

[7] A. Vouilloz, et al., JSSC, vol. 36, pp. 440-451, 2001.

[8] H. C. Nathanson, et al., IEEE Trans. on Electron Devices, vol. 14, pp. 117-133, 1967.

[9] W.-T. Hsu, et al., Proceedings, ISSCC, 2006, pp. 1111-1120.

[10] M. Akgul, et al., "A Negative Capacitance Equivalent Circuit Model for Parallel-Plate...", IEEE Trans. on UFFC, In Press.

[11] T. O. Rocheleau, et al., Proceedings, IEEE Int. Conf. on MEMS, 2014, pp. 210-213.

[12] W.-C. Li, et al., Proceedings, Transducers, 2013, pp. 24692472.

Contact: Tristan O. Rocheleau, tel:+1-607-232-2162; tristan@, eecs.berkeley.edu 